\title{
COMPUTATIONAL HYDRODYNAMIC ANALYSIS OF A HIGHLY SKEWED MARINE PROPELLER
}

\author{
Kadda Boumediene $^{1}$, S.E Belhenniche ${ }^{2}$, Omar Imine ${ }^{2}$. Mohamed Bouzit ${ }^{3}$ \\ ${ }^{1}$ Mechanical Engineering Department, University of Sciences and Technology of Oran (U.S.T.O), Algeria; \\ E-mail: kadda.boumediene@univ-usto.dz \\ ${ }^{2}$ Marine Engineering Department, University of Sciences and Technology of Oran (U.S.T.O), Algeria. \\ ${ }^{3}$ Mechanical Engineering Department, University of Sciences and Technology of Oran (U.S.T.O), Algeria.
}

\begin{abstract}
:
The objective of the current paper is to study the flow around Seiun Maru Highly Skewed (HSP) marine propeller by assessment of blade forces and moments under non-cavitating case. The calculations are performed in open water (steady case) and non-uniform ship wake (Unsteady case). The governing equations based on Reynolds Averaged Navier-Stokes Equation (RANSE) are solved using Finite Volume Method. Ansys Fluent 14.0 is used to implement the simulation. For the steady case, Moving Reference Frame $(M R F)$ is selected while sliding mesh technique is adopted for the unsteady case. Calculated open water performances in terms of thrust and torque coefficients fit very well with experimental data for a wide range of advance ratio. In the unsteady calculations, axial velocities, deduced from the nominal wake, are introduced in the Ansys fluent code. To locate suitably the non-uniform wake in the propeller front plane, three positions of inlet wake have been taken into account to determine their effects on the accuracy of the results. Obtained results show that computed performances are improved compared to panel method when the inlet is close to the propeller.
\end{abstract}

Keywords: Seiun Maru highly skewed propeller, steady, unsteady, RANS, sliding mesh technique, MRF.

\begin{tabular}{llll}
\hline NOMENCLATURE & & \\
$\boldsymbol{D}$ & Propeller diameter & $\boldsymbol{J}$ & Advance coefficient \\
$\boldsymbol{n}$ & Propeller rotational revolution & $\boldsymbol{A}_{\boldsymbol{e}} / \boldsymbol{A}_{\mathbf{0}}$ & Expanded Area Ratio \\
$\boldsymbol{V}_{\mathbf{0}}$ & Ship speed & $\emptyset(\mathbf{i})$ & Geometric pitch angle \\
$\boldsymbol{V}_{\boldsymbol{a}}$ & Advance velocity & $\overline{\boldsymbol{\rho} \boldsymbol{u}_{\boldsymbol{t}}^{\prime} \boldsymbol{u}_{\boldsymbol{J}}^{\prime}}$ & Unknown Reynolds Stresses \\
$\boldsymbol{T}$ & Propeller thrust & $\boldsymbol{u}_{\boldsymbol{i}}, \boldsymbol{u}_{\boldsymbol{j}}$ & Time average velocity \\
$\boldsymbol{Q}$ & Propeller torque & $\boldsymbol{P}$ & Time average pressure \\
$\boldsymbol{K}_{\boldsymbol{T}}$ & Propeller thrust coefficient & $\boldsymbol{\mu}$ & Dynamic viscosity \\
$\boldsymbol{K}_{\boldsymbol{Q}}$ & Propeller torque coefficient & $\boldsymbol{k}$ & Kinetic energy \\
$\boldsymbol{\eta}_{\mathbf{0}}$ & Propeller efficiency & $\boldsymbol{\mu}_{\boldsymbol{t}}$ & Eddy Viscosity \\
$\boldsymbol{C}_{\boldsymbol{p}}$ & Pressure coefficient & $\boldsymbol{G}_{\boldsymbol{\omega}}$ & Generation of $\boldsymbol{\omega}$ \\
$\boldsymbol{\rho}$ & Water density & $\boldsymbol{Y}_{\boldsymbol{k}}$ & Dissipation of $\boldsymbol{k}$ due to turbulence \\
$\boldsymbol{r}$ & Propeller radius at each section & $\boldsymbol{Y}_{\boldsymbol{\omega}}$ & Dissipation of $\boldsymbol{\omega}$ due to turbulence \\
$\boldsymbol{R}$ & Propeller radius & $\boldsymbol{S}_{\boldsymbol{k}}, \boldsymbol{S}_{\boldsymbol{\omega}}$ & User-defined source terms \\
$\boldsymbol{R} \boldsymbol{e}$ & Reynolds number & $\boldsymbol{D}_{\boldsymbol{\omega}}$ & Cross-diffusion term \\
$\boldsymbol{Z}$ & Blades number & $\boldsymbol{G}_{\boldsymbol{k}}$ & Generation of turbulence kinetic energy due to \\
$\boldsymbol{w}$ & Wake fraction & & mean velocity gradients
\end{tabular}

\section{Introduction}

Propeller runs behind a highly turbulent wake in the stern of the vessel. Thus, the produced chaotic region of flow causes vibratory forces essentially on the propeller which are transferred to the ship hull directly through the shaft-line. Of course, these vibrations disturb both crew and passengers. Although it is extremely difficult to predict propeller performances operating in the vessel's hull stern (2009), large researches based on experiments and numerical methods are devoted for this propose in both open water and behind a ship stern. In the recent years, many numerical methods, based on solving Reynolds-Averaged Navier-Stokes equations, are carried out leading to remarkable progress in order to determine the fluid flow around marine propellers. 
For example, Watanabe et al. (2003) used numerical simulations for steady and unsteady non-cavitating case around conventional Seiun Maru propeller. The obtained results of hydrodynamic performances such as thrust and torque coefficients were in a good agreement with the measurements for a wide range of advance coefficient. Rhee et al. (2005) used Navier-Stokes solver for the validation of flow around P5168 marine propeller using unstructured hybrid mesh. The computation hydrodynamic characteristics results were getting closer to the measured values.

Mossad et al. (2011) achieved a numerical calculation to determinate the open water characteristics of DTMB-P4119 marine propeller by using RANS method combined with a validation of two turbulence models $k-\varepsilon$ and $k-\omega$, the computational results indicate good agreement with the experimental data. Prakash and Nath (2012) performed a numerical simulation to estimate the open water characteristics of four bladed Wageningen B4.55 propeller by adopting an unstructured mesh and standard $k-\varepsilon$ turbulence model. The thrust and torque coefficients were evaluated by using regression equation. The differences between numerical and measurable results are significant due probably to the use of instructed mesh. Wang et al. (2010) create a mathematical model to investigate the effect of boundary conditions on propeller's characteristics. The calculation of open water performance of DTMB 4119, 4382, and 4384 were performed and the obtained results are in good agreement with the experimental results.

Boumediene and Belhenniche (2016) performed a numerical analysis to predict the hydrodynamic performances of DTMB 4119 marine propeller. The predicted values of torque and thrust coefficients, obtained by selecting $k-\omega$ model in the numerical simulation were found in good accordance with the experimental results. Belhenniche et al. (2016) studied the effect of blade number, pitch ratio and expanded area on the hydrodynamic performances in open water using RANS Solver. This parametric study reveals that the hydrodynamic performance of four bladed propeller are superior compared with other configurations.

The paper presents a numerical simulation of steady and unsteady turbulent flow in non-cavitating case around the Seiun Maru HSP propeller. The main purpose of this work is to reproduce accurately the hydrodynamic characteristics such as thrust, torque and pressure coefficients in open water and behind a ship hull. It is emphasized in this study on the effect of nominal wake position on the propeller thrust. Indeed, in most numerical researches, different distances of the inlet from the propeller plan are adopted without giving justification (Ji et al., 2011, Belhenniche et al. and 2016, Watanabe et al. 2003). Therefore, a numerical calculation is conducted to define the best position of the nominal wake by using three distances from the propeller plan. The obtained results are compared with panel method.

\section{Propeller Characteristics}

The Seiun Maru HSP propeller is chosen as a reference case for the CFD benchmark exercise; in cavitating and non-cavitating cases. HSP propeller is a five bladed, highly skewed, variable pitch and right-handed and originally designed in Japan. The main characteristics of this propeller are shown in Table 1 and its geometry in Fig. 1.

Table 1: Main characteristics of the Seiun Maru HSP propeller.

\begin{tabular}{|l|c|}
\hline \multicolumn{1}{|c|}{ Propeller Full Scale } & HSP \\
\hline Number of Blades $(Z)[-]$ & 5 \\
\hline Diameter $(D)[\mathrm{m}]$ & 3.6 \\
\hline Boss Ratio $(r / R)[-]$ & 0.1972 \\
\hline Blade Thickness Ratio $[-]$ & 0.0496 \\
\hline Pitch ratio $(P(i) / D)[-]$ & Variable \\
\hline Expanded Area Ratio $\left(A_{e} / A_{0}\right)[-]$ & 0.7 \\
\hline Rake $\left[{ }^{\circ}\right]$ & -3.03 \\
\hline Skew at $R[\mathrm{~m}]$ & 1.3367 \\
\hline Blade Section & Modified SRI-B \\
\hline Design advance coefficient $(J)[-]$ & 0.85 \\
\hline Material $[-]$ & AlBC3 (Ni-Al-Bz) \\
\hline
\end{tabular}



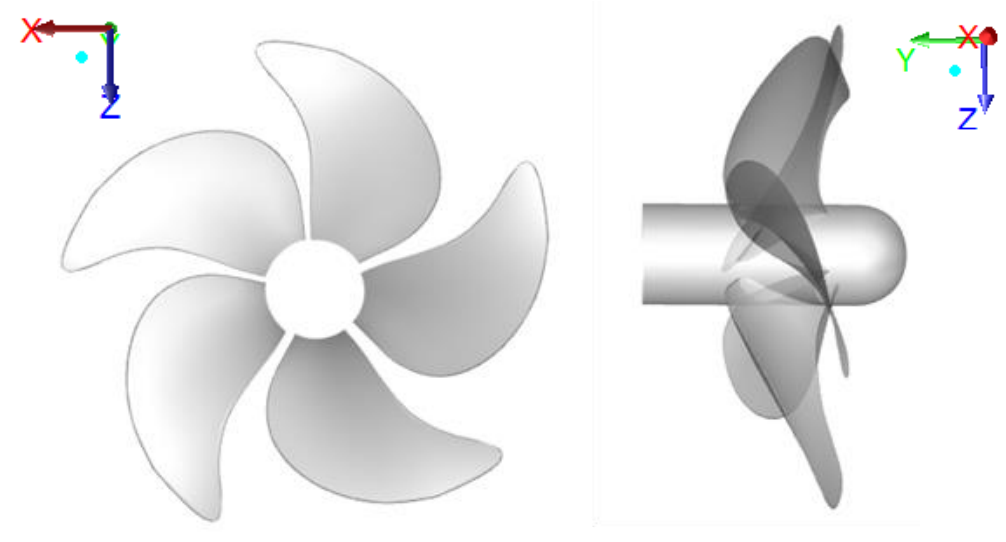

Fig. 1: Geometry of the Seiun Maru HSP propeller.

\section{Propeller Drawing Procedures}

To generate the geometry of the propeller, a Fortran program was developed based on the geometric characteristics of Seiun Maru HSP Propeller. This program provides the spatial point coordinates for each radial section. The produced vertices are moved by appropriate skew and rake values respectively in $\mathrm{x}$ and $\mathrm{y}$ directions to get the expanded sections. Then, all sections are deflected by geometric pitch angle which depends on the pitch value and the radial position of the section (Carlton, 2018 and Bertram, 2012). Finally, trigonometric projections are used to obtain projected sections. All equations on which geometry is based on are described below:

The geometric pitch angle is given by:

$\varnothing(i)=\operatorname{arctg}(P(i) / 2 \pi r(i))$

Where $P(i)$ is the blade pitch distribution.

Transformations on profile coordinates operated by skew and rake distribution are given by:

$x p(i)=x p(i)+\left(\left(\frac{C(i)}{2}\right)+\operatorname{Skew}(i)\right)$

And

$y p(i)=y p(i)+\operatorname{Rake}(i)$

Where $C(i)$, Skew $(i)$ and Rake $(i)$ are respectively chord, skew and rake distributions.

Finally, coordinate equations for the projected profile are:

$$
\begin{aligned}
& x(i)=x p(i) \cdot \cos (\varnothing)-y p(i) \cdot \sin (\varnothing) \\
& y(i)=x p(i) \cdot \sin (\varnothing)+y p(i) \cdot \cos (\varnothing) \\
& z(i)=r(i) \cdot \cos \alpha(i)
\end{aligned}
$$

Therefore, spatial points' coordinates of each section are exported to preprocessor Gambit describing a shape of propeller blade. Appropriate points are connected into curves by using spline function to create faces and blade volume.

The shaft is also connected to the propeller root blades by using T-junction sequence on Gambit. Fig. 2 shows the propeller drawing steps. 


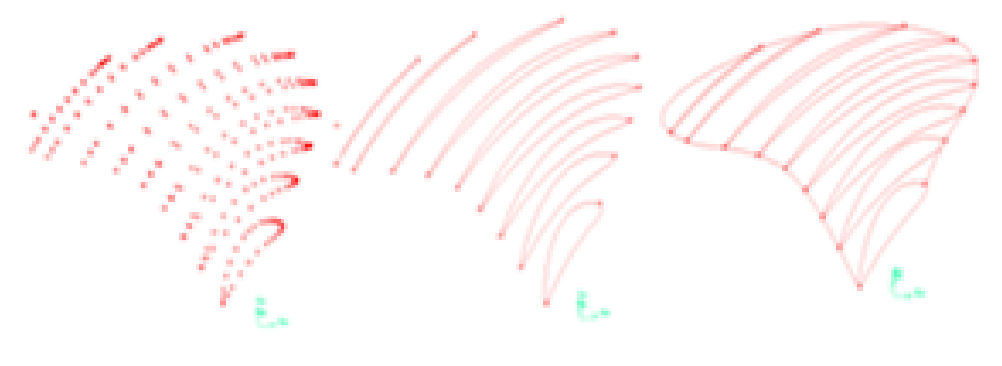

Fig. 2: The propeller drawing steps.

\section{Mesh Generation and Boundary Conditions}

Fig. 3 shows the propeller surface mesh with using an unstructured mesh in Gambit. For both steady and unsteady cases, the same mesh was adopted. First, the blade surface was meshed with small constant triangle cells with the size is approximately $0.0055 \mathrm{D}$ in all blade faces. The shaft surface was meshed with triangles cells of $0.0198 \mathrm{D}$.

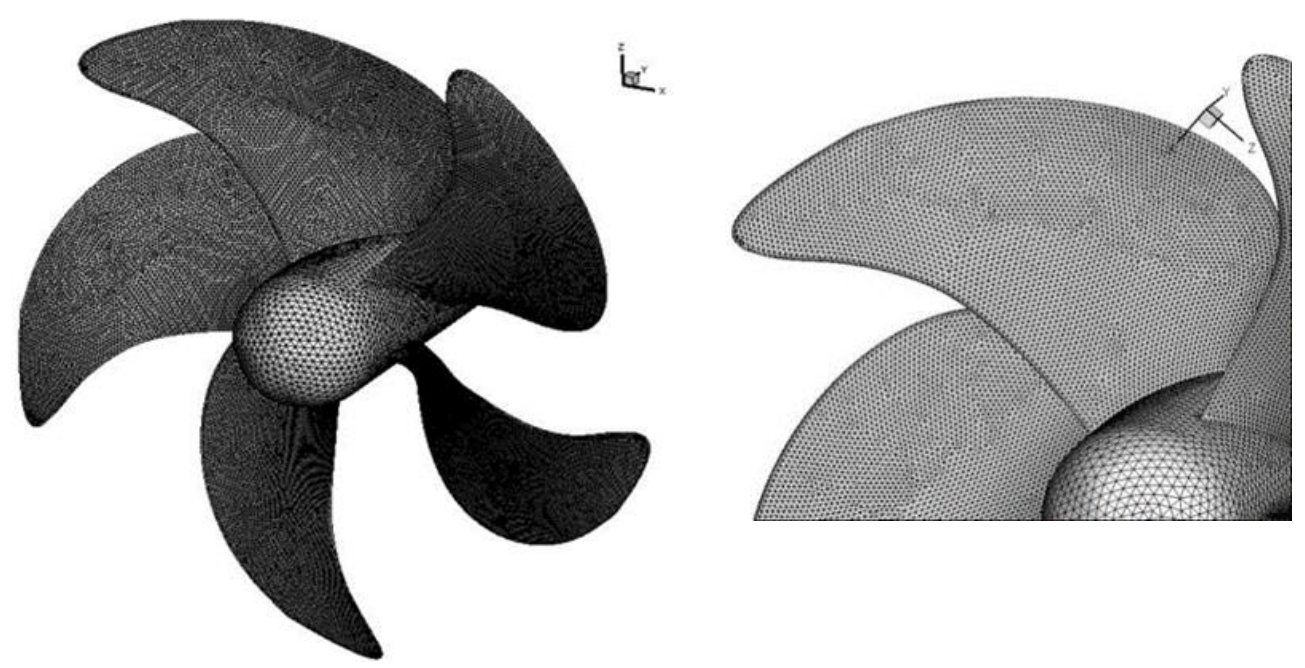

Fig. 3: The propeller surface mesh.

All five blades of the propeller were modeled to simulate the flow in open water and non-uniform ship wake. The computational domain was split into inner and outer blocks. For the steady case, flow equations are solved by adopting a rotating frame linked to the propeller and all blocks are also considered as rotary. In Fluent code, this procedure is called Moving Reference Frame (MRF) as reported by Kaewkhiaw (2018) and Kinaci et al. (2018).

However, for the unsteady cases, the wake which is considered as the inlet of calculation domain is non-homogeneous. Therefore, only Sliding Mesh Technique is available in the fluent code to deal with this case. It consists to operate automatically a rotation of calculation domain by step of 1 degree repeatedly until the convergence of the simulation, which is called external loop. At each step, the flow equations are solved giving propeller thrust and torque at the end of the convergence named internal loop. The simulation convergence is achieved when the forces signal become periodic (Seo et al., 2010). Practically, two inner blocs are considered rotational and the rest is taken as stationary blocs. The six cylinder blocs are connected between them using interfaces. A hybrid local mesh was used to control the mesh size, tetrahedral cells for the inner blocs and hexahedral for the outer blocks.

For the simulation of steady case, the inlet boundary is at $1.5 \mathrm{D}$, the outlet is at $3.5 \mathrm{D}$, and the outer boundary is at $1.4 D$ from the shaft axis (see Fig. 4). Fig. 5 illustrates the mesh generation at cross section of the full domain. For the unsteady calculations, three cases were considered by changing the inlet position respectively at $0.4 D, 1 D$ and $2 D$ while the outlet and the outer boundary are positioned respectively at $7 D$, and $3 D$ as it is illustrated in Fig. 6. 
The outlet condition is set as a static pressure, the outer boundary is characterized by a slip condition and the no slip condition was imposed for the blade and hub surfaces. The fluid is considered as rotational around the y-axis (shaft axis). The only difference between the two cases is at the inlet condition; uniform that depends on the advance coefficient $J$ for the steady case, and non-uniform chart of axial velocities $V_{a}$ measured by Ukon et al. (1991, 1989) and Kurobe et al. (1983).
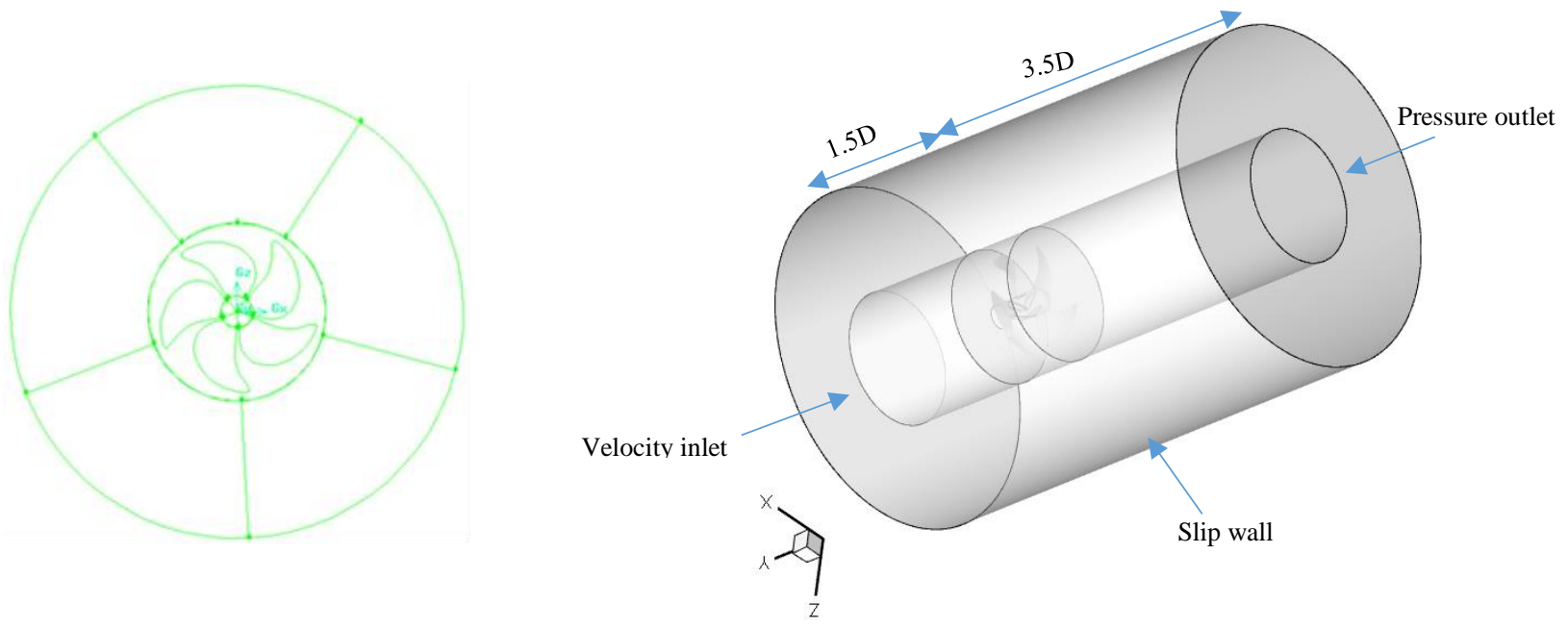

Fig. 4: Computational domain for the steady case.

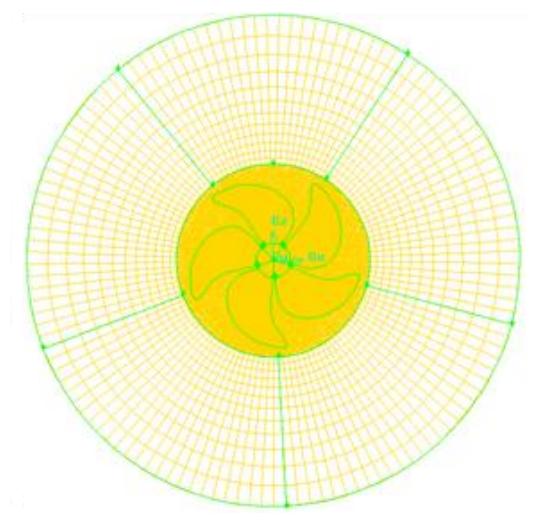

Fig. 5: Mesh generation for the steady simulation.

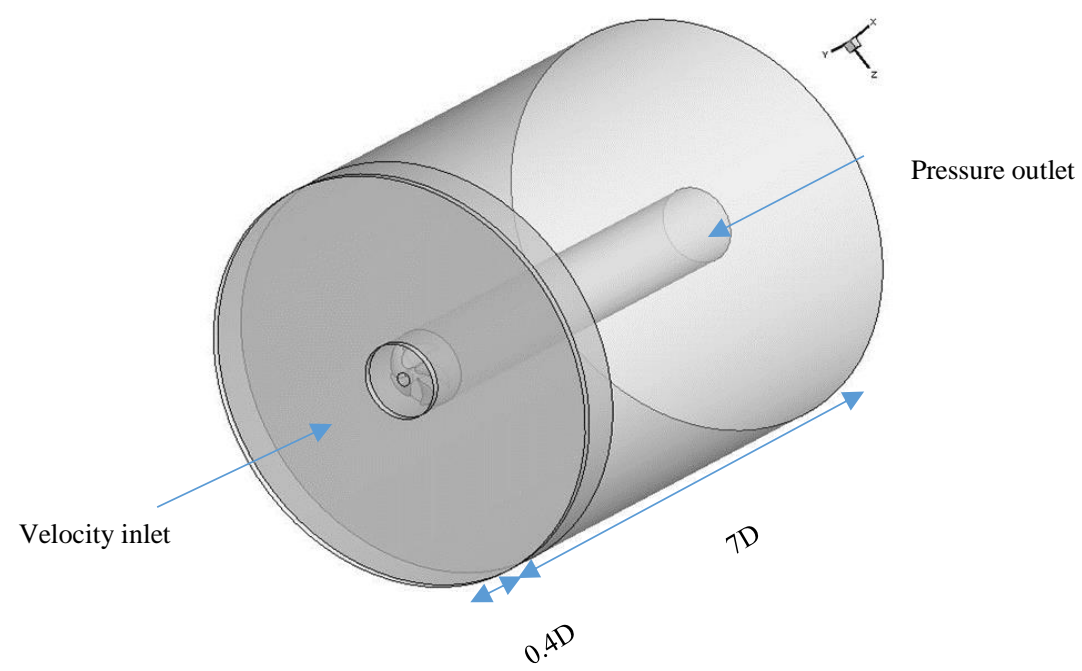

Fig. 6: Computational domain for the unsteady case $\left(1^{\text {st }}\right.$ case $)$. 


\section{Turbulence Modeling and Numerical Procedure}

The continuity and the momentum equations are described as follows:

$$
\begin{aligned}
& \frac{\partial u_{i}}{\partial x_{i}}=0 \\
& \frac{\partial\left(\rho u_{i}\right)}{\partial t}+\frac{\partial\left(\rho u_{i} u_{j}\right)}{\partial x_{j}}=-\frac{\partial P}{\partial x}+\frac{\partial}{\partial x_{j}}\left(\mu \frac{\partial u_{i}}{\partial x_{j}}-\overline{\rho u_{\imath}^{\prime} u_{\jmath}^{\prime}}\right)
\end{aligned}
$$

\subsection{Turbulence modeling}

The turbulence $k-\varepsilon$ (Launder and Spalding, 1972) and $k-\omega S S T$ (Menter, 1994) models are selected; their equations are given as follows:

$$
\begin{gathered}
\boldsymbol{k}-\boldsymbol{\varepsilon} \text { Model: } \\
\frac{\partial}{\partial t}(\rho k)+\frac{\partial}{\partial x_{i}}\left(\rho k u_{i}\right)=\frac{\partial}{\partial x_{j}}\left[\left(\mu+\frac{\mu_{t}}{\sigma_{k}}\right) \frac{\partial k}{\partial x_{j}}\right]+G_{k}-\rho \varepsilon \\
\frac{\partial}{\partial t}(\rho \varepsilon)+\frac{\partial}{\partial x_{i}}\left(\rho \varepsilon u_{i}\right)=\frac{\partial}{\partial x_{j}}\left[\left(\mu+\frac{\mu_{t}}{\sigma_{\varepsilon}}\right) \frac{\partial \varepsilon}{\partial x_{j}}\right]+C_{1 \varepsilon} \frac{\varepsilon}{k} G_{k}-\rho C_{2 \varepsilon} \frac{\varepsilon^{2}}{k} \\
\boldsymbol{k}-\boldsymbol{\omega} \boldsymbol{S S T} \text { Model: } \\
\frac{\partial}{\partial t}(\rho k)+\frac{\partial}{\partial x_{i}}\left(\rho k u_{i}\right)=\frac{\partial}{\partial x_{j}}\left[\left(\mu+\frac{\mu_{t}}{\sigma_{k}}\right) \frac{\partial k}{\partial x_{j}}\right]+G_{k}-Y_{k}+S_{k} \\
\frac{\partial}{\partial t}(\rho \omega)+\frac{\partial}{\partial x_{i}}\left(\rho \omega u_{i}\right)=\frac{\partial}{\partial x_{j}}\left[\left(\mu+\frac{\mu_{t}}{\sigma_{\omega}}\right) \frac{\partial \omega}{\partial x_{j}}\right]+G_{\omega}-Y_{\omega}+D_{\omega}+S_{\omega}
\end{gathered}
$$

Explicit details for the models can be found in the literature of Launder and Spalding (1972) and Menter (1994).

\subsection{Numerical Procedure}

Segregated solvers Simple and Piso as velocity-coupling algorithms were chosen respectively for both steady and unsteady simulations. Quick scheme was adopted for the discretization of diffusion and convection terms of momentum equations and Standard scheme was chosen to discretize the continuity equation. Finally, upwind scheme was selected for the discretization of the equations of turbulent energy and dissipation. Related factors of relaxation were adjusted from the default values during the simulation. The condition of non-uniform inflow was introduced in Ansys Fluent code by reading a profile of axial velocities with their appropriate Cartesian coordinates

\section{Test Conditions}

The measurement of wake distribution on the propeller plane in the full load condition of the Seiun- Maru Ship model was carried out by Mitsui Engineering and Shipbuilding laboratory large tank by Ukon et al. (1989). The results are available for a ship model speed of 9 knots. Vector velocity contours and iso-axial velocity lines are shown in Fig. 7.

The numerical computations were achieved on a PC with 64-bit processor, Intel (R) Core (TM) i7-3770 CPU@ $3.40 \mathrm{GHz}$, and $8 \mathrm{~GB}$ RAM. To match the conditions of the experiment (Ukon, 1989) in open water tests, the simulation for the steady case was carried out for a Seiun Maru model with a scale of 1/9 given a diameter value of $D=0.4 \mathrm{~m}$. The propeller angular velocity is maintained constant during tests with a value of $n=3.63 \mathrm{rps}$ corresponding to $R_{e}=5.8 \times 10^{5}$. In this case, the processing time for calculation is around 7 hours for each advance coefficient. While for the unsteady case, the simulation is carried out for full-scale propeller with a diameter equal to $3.6 \mathrm{~m}$ and propeller revolution of $90.7 \mathrm{rpm}$. The ship speed considered in this case is 9 knots giving an advance 
parameter of 0.85 . All computations are carried out by opting for a value of time step equal to $0.00183756 \mathrm{~s}$ which corresponds to a rotation angle of one degree.

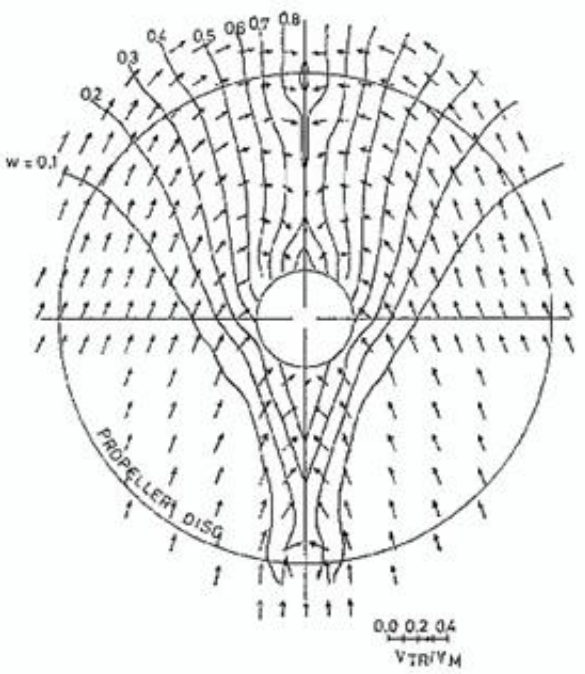

Fig. 7: Wake distribution of the Seiun- Maru ship model.

\section{Results and Discussion}

\subsection{Open water characteristics}

The convergence history of the simulation results for advance coefficient $J=0.4$ are shown in Fig. 8 . Fig. 9 shows the calculated open water characteristics compared with the experiments carried out by Ukon et al. $(1991,1989)$ and Kurobe et al. (1983). The red lines show the predicted results, and the green points are the measured results. As it can be seen, the computational results indicated well agreement with experimental results. In Table 2, a direct comparison of the computational results with experimental data has been carried. The average error of thrust is $4.18 \%$ and $6.04 \%$ for torque coefficients. For high values of advance coefficient, the deviation of thrust increase as it is confirmed by Wang et al. (2010).

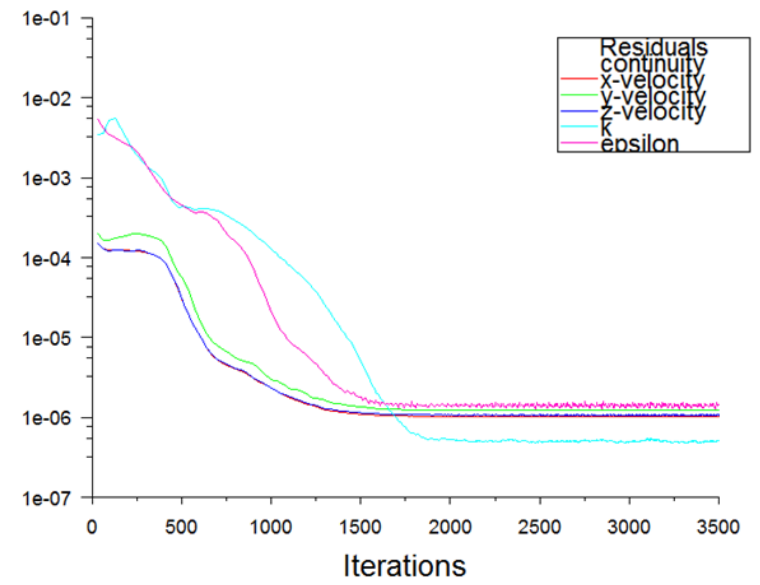

Fig. 8 Residuals history with the number of iterations for $J=0.4$.

Fig. 10 illustrates the $C_{p}$ contours on the suction side for different advance coefficient $J=0.3,0.5,0.7$ and 0.9 . It is clearly seen that the maximal values of $C_{p}$ are shown on the leading edge excepting the tip of the blade where it becomes minimal due to the tip vortex. The suction region is more visible for low advance coefficient due to the decrease of incidence angle. It is noticed that the pressure contour is the same for the five blades ensuring perfectly the periodicity of the flow. 
Table 2: Open water performances of the HSP marine propeller.

\begin{tabular}{|l|c|c|c|c|c|l|}
\hline$J$ & $K_{T}$ & $K_{T}$ & Error & $10 K_{Q}$ & $10 K_{Q}$ & Error $\%$ \\
\hline Exp & $R A N S$ & $\%$ & $E x p$ & $R A N S$ & \\
\hline 0.1 & 0.4400 & 0.4176 & 5.09 & 0.5890 & 0.5700 & 3.22 \\
\hline 0.3 & 0.4014 & 0.3891 & 3.06 & 0.5500 & 0.5300 & 3.63 \\
\hline 0.4 & 0.3086 & 0.3099 & 0.42 & 0.4500 & 0.4597 & 2.15 \\
\hline 0.5 & 0.2586 & 0.2696 & 4.25 & 0.3900 & 0.4120 & 5.64 \\
\hline 0.6 & 0.2100 & 0.2234 & 6.38 & 0.3330 & 0.3590 & 7.80 \\
\hline 0.7 & 0.1600 & 0.1743 & 8.94 & 0.2800 & 0.3003 & 7.25 \\
\hline 0.8 & 0.1006 & 0.1218 & ------- & 0.2200 & 0.2359 & 7.22 \\
\hline 0.9 & 0.0510 & 0.0648 & ------- & 0.1400 & 0.1639 & 17.07 \\
\hline & Average Error & $4.18 \%$ & Average Error & $6.04 \%$ \\
\hline
\end{tabular}
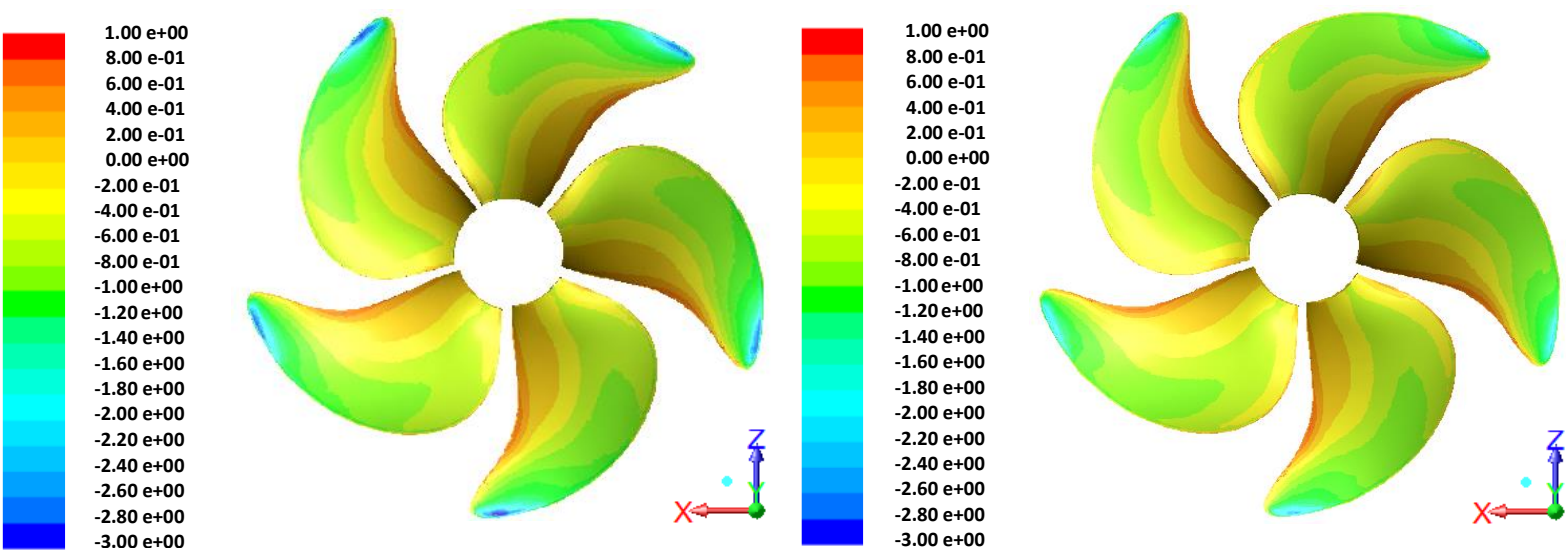

$$
J=0.3
$$
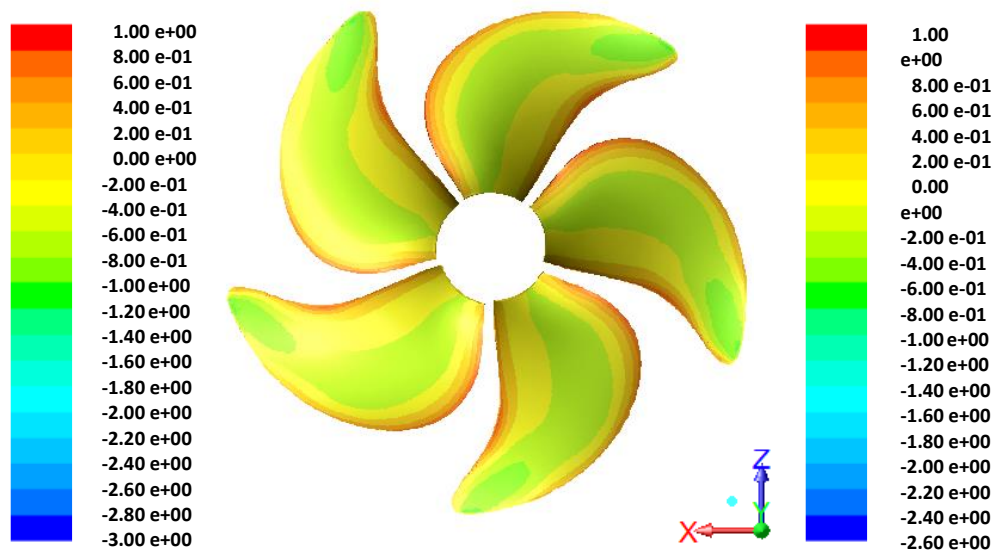

$J=0.7$

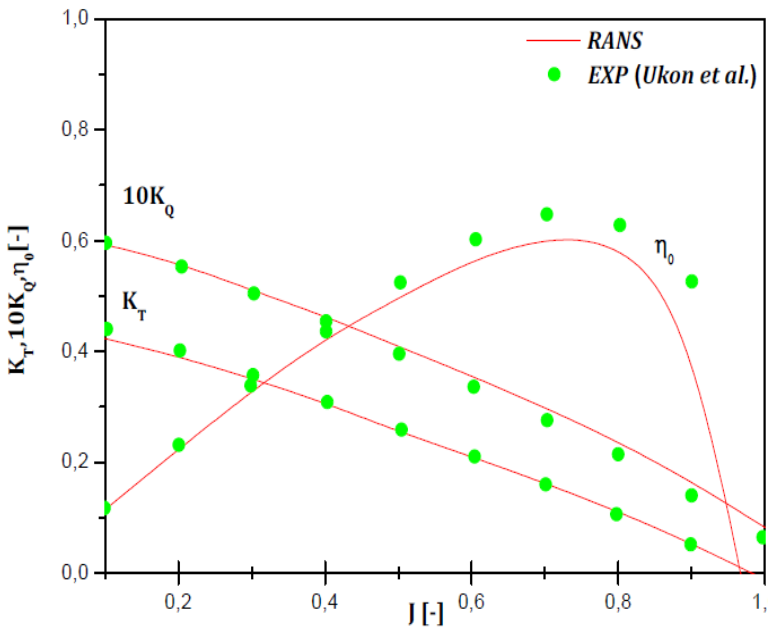

Fig. 9: Open water characteristics of HSP marine propeller.

Fig. 10: $C_{p}$ contours on the suction side of HSP marine propeller. 


\subsection{Non-uniform ship wake}

Fig. 11 shows the thrust coefficient $K_{T}$ for one and five blades during one revolution (for different inlet positions) and the convergence history of the simulation for the first position. The Green points are the Nakatake et al. (2002) results and the reds are calculated results. The Seiun Maru HSP averaged thrust coefficient $K_{T}$ is 0.188 compared with Nakatake results. It is observed in Table 3 that the inlet position corresponding to $0.4 D$ exhibits the smallest error estimated to $3.79 \%$. Therefore, it is necessary to locate the inlet position near the propeller front plan to avoid loss in thrust.

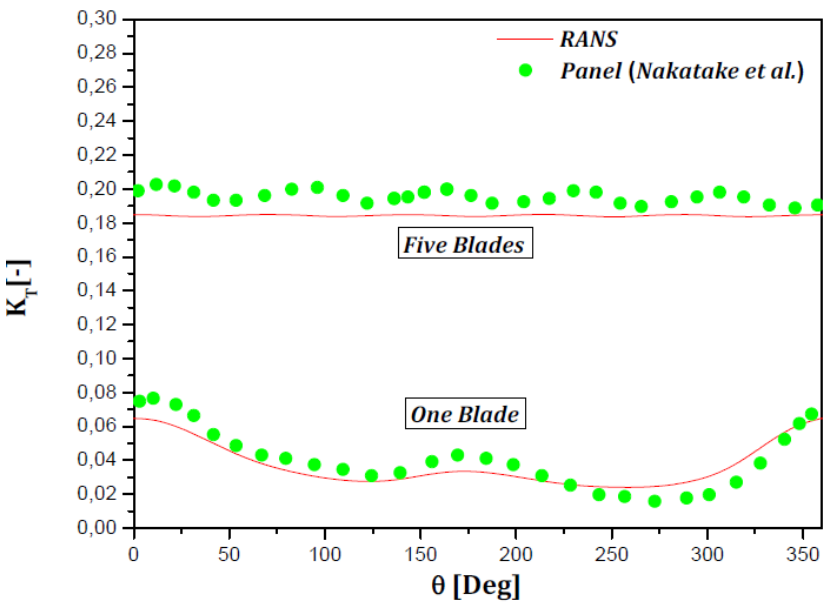

(a)

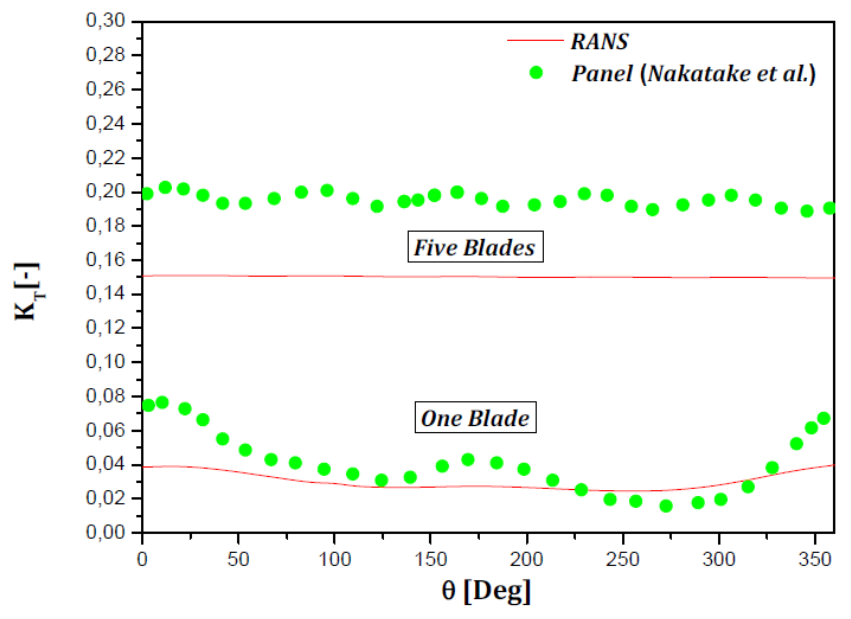

(c)

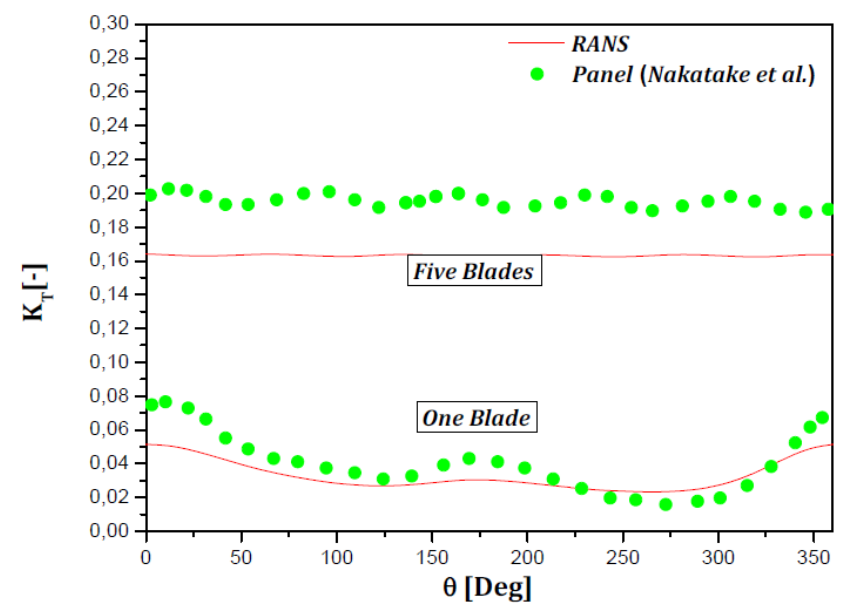

(b)

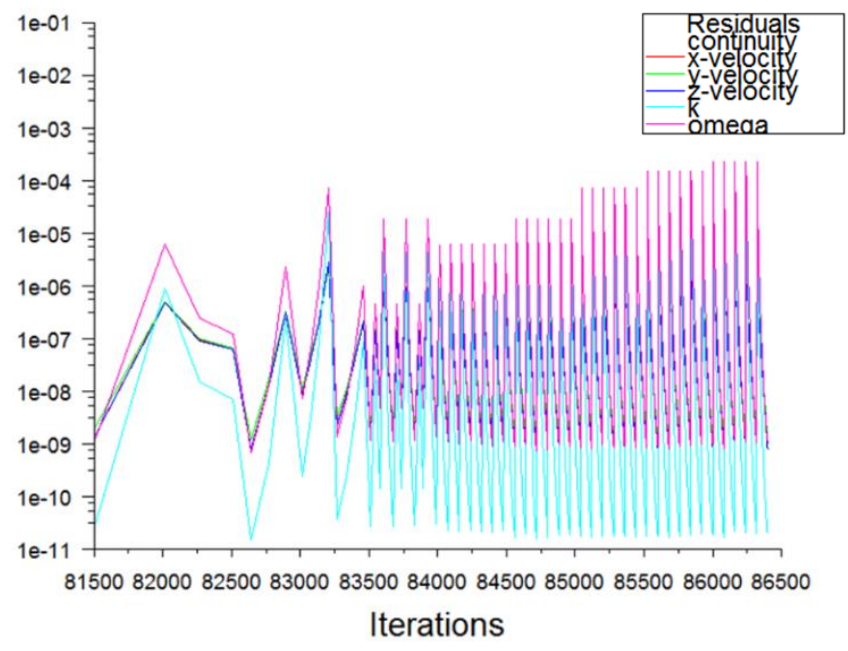

(d)

Fig. 11: Variation of $K_{T}$ during propeller rotation: (a) First position, (b) Second position, (c) Third position (d) The convergence history of the simulation for the first position.

Fig. 12 shows the pressure distribution on the blade suction and pressure sides at $0^{\circ}$ and $180^{\circ}$ degrees for all inlet position cases. It is clear that pressure contours change depending on the blade position due to the non-uniform inflow. At the top position, where the non-uniform wake exhibits an important lack of axial velocity, the peak of the negative pressure on the backside is largest leading to the occurrence of cavitation. The exam of pressure contours shows that the suction side is more pronounced for the case where the inlet is located at $0.4 D$. This confirms the reason why the calculated average thrust is the most higher compared to other positions.

Table 3: Average $K_{T}$ for different positions.

\begin{tabular}{|l|l|l|l|l|}
\hline & First position $(0.4 D)$ & Second position $(1 D)$ & Third position $(2 D)$ & Panel method \\
\hline Average $K_{T}$ & 0.188 & 0.163 & 0.150 & 0.195 \\
\hline Error & $3.79 \%$ & $16.53 \%$ & $23.08 \%$ & ----- \\
\hline
\end{tabular}



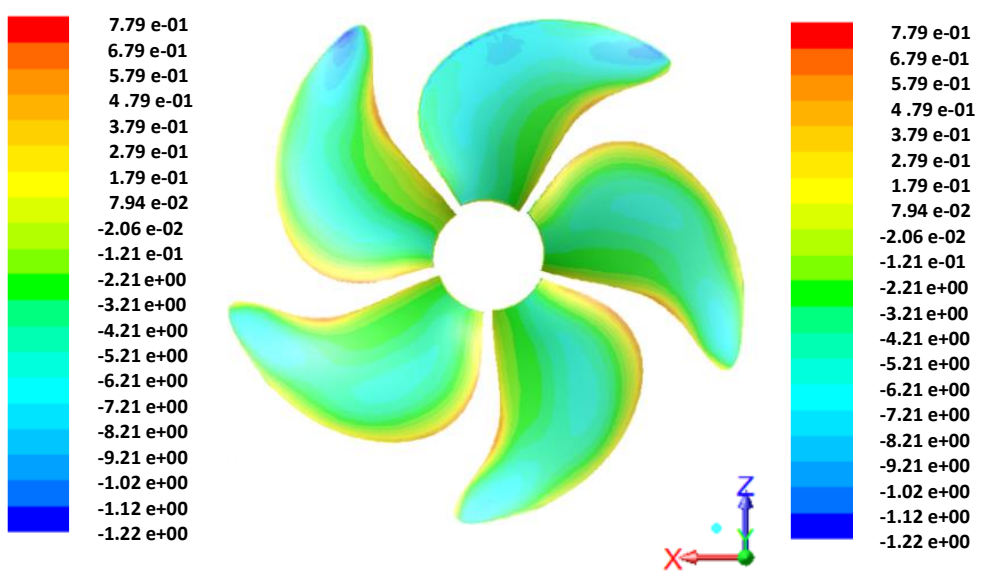

(a) $\theta\left[0^{\circ}\right]$
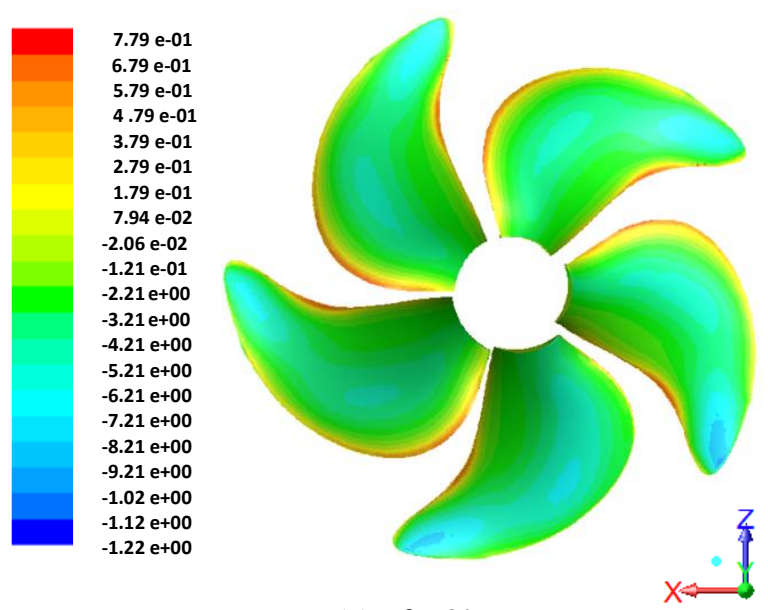

(c) $\theta\left[0^{\circ}\right]$
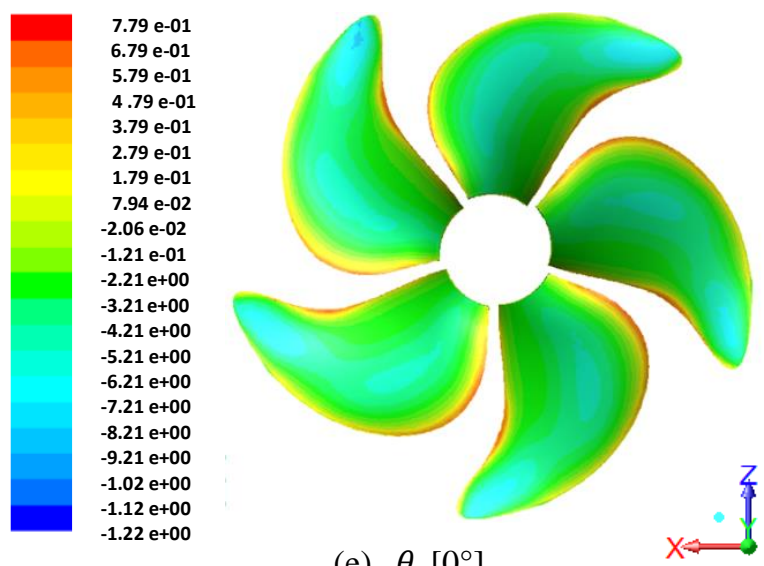

(e) $\theta\left[0^{\circ}\right]$

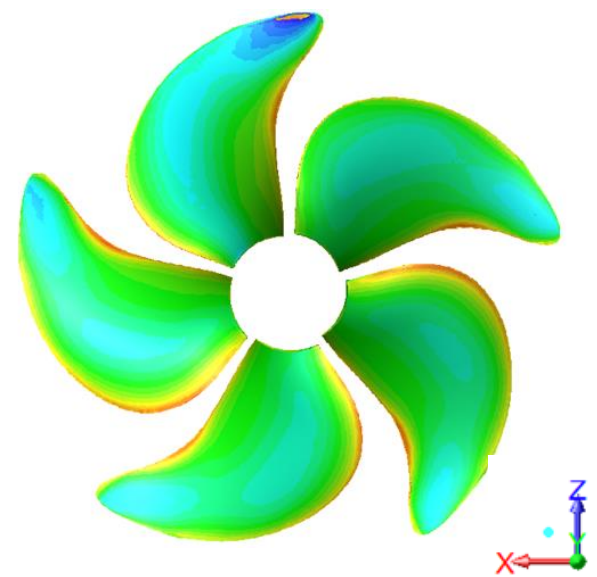

(b) $\theta\left[180^{\circ}\right]$

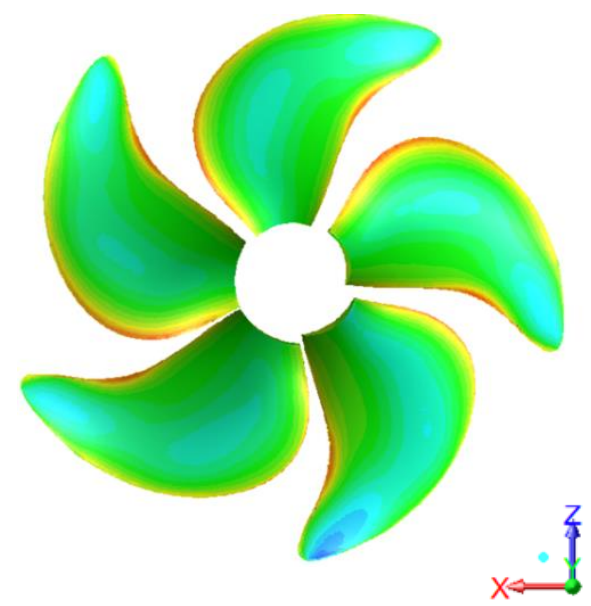

(d) $\theta\left[180^{\circ}\right]$

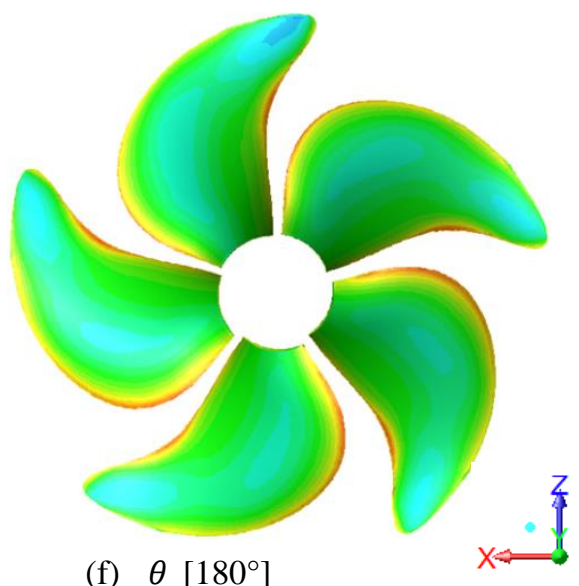

Fig. 12: Pressure coefficient distribution during propeller rotation: first position (a \& b), second position (c \& d), third position (e \& f).

Fig. 13 shows the spiral streamlines released from propeller after three revolutions in the case of non-uniform ship wake calculation. It can be observed that no flow separation exists on the whole propeller which means that attack angle no exceed the stall angle on the blades. It is also noted that the recorded acceleration of flow over the suction side near the tip is due essentially to the increase of tangential velocity blade. 

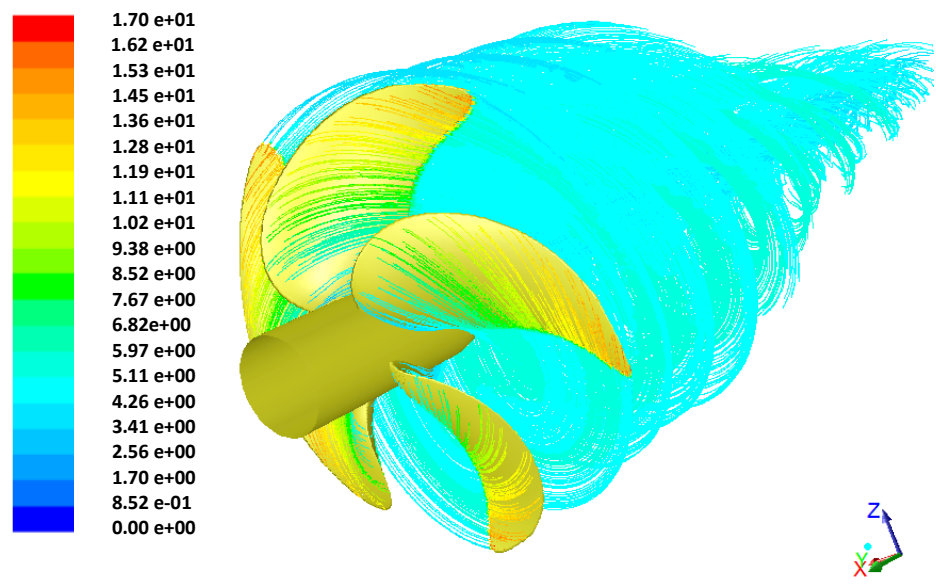

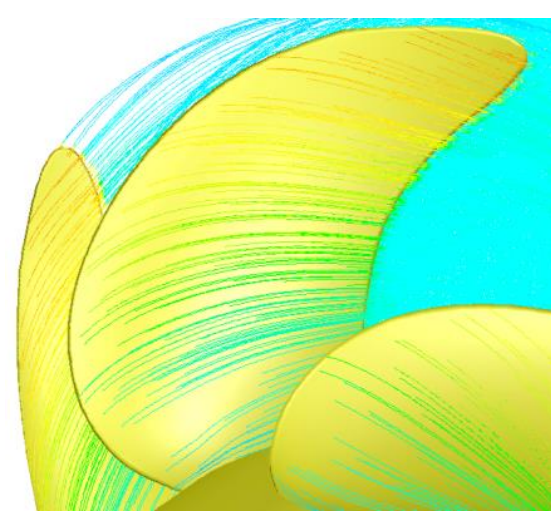

Fig. 13: Streamlines around propeller blades (first position).

\section{Conclusion}

In this study, numerical simulations are performed to determine HSP Seiun Maru propeller characteristics in the case of open water and non-uniform ship wake by using a CFD software, Ansys Fluent.

In the first part, where the steady case is considered, results of numerical calculations show a good agreement with experimental measurements and the average error of $K_{T}$ was estimated by $4.18 \%$ while the average error of $K_{Q}$ was $6.04 \%$ for the investigated interval of advance coefficient.

In the second part which is devoted to the case of non-uniform ship wake, the flow is assumed unsteady during propeller revolution. Computational results show that the positioning of the inlet boundary influence considerably the accuracy of results in term of thrust coefficient. To ensure a good estimation of this coefficient, it is recommended to bring closer the inlet boundary to the propeller. Indeed, the average of $K_{T}$, calculated during propeller revolution, agrees well with that by panel method for the case of $0.4 D$ inlet location with an error of 3.79 $\%$. Additionally, qualitative results, characterized by pressure contours on the blade, confirm this tendency. It is important to note that non-uniform wake distribution is determined without the presence of the propeller at reference plane precisely.

\section{Acknowledgements}

ANSYS Fluent software used in this study is supported by the laboratory of composite and innovative materials, Mechanical Engineering Department, University of Sciences and Technology of Oran (U.S.T.O), Algeria.

\section{References}

Belhenniche, S. E., Aounallah, M., Imine, O., and Çelik, F. (2016): Effect of geometric configurations on hydrodynamic performance assessment of a marine propeller, Brodogradnja /Shipbuilding Journal, Vol. 67, No. 4. https://dx.doi.org/10.21278/brod67403

Bertram, V. (2012): Practical Ship Hydrodynamics ( $2^{\text {nd }}$ Edition), Elsevier Ltd. https://dx.doi.org/10.1016/C2010-0-68326-X

Boumediene, K., and Belhenniche, S. E. (2016): Numerical analysis of the turbulent flow around DTMB 4119 marine propeller, International Journal of Marine and Environmental Sciences. Vol. 10, No. 2.

http://dx.doi.org/10.5281/zenodo.1111913

Carlton, J. (2018): Marine Propeller and Propulsion (4 ${ }^{\text {th }}$ Edition), Elsevier Ltd., 609 pp.

https://dx.doi.org/10.1016/C2014-0-01177-X

Ghassemi, H. (2009): The effect of wake flow and skew angle on the ship propeller performance, Transaction B: Mechanical Engineering. Vol. 16, No. 2, pp. 149-158. 
Ji, B., Wang, X., Peng, X., Wu, Y. and Xu, H. (2011): Unsteady numerical simulation of cavitating turbulent flow around a highly skewed model marine propeller, Journal of Fluids Engineering, Vol. 133 / 011102-3.

https://doi.org/10.1115/1.4003355

Kaewkhiaw, P. (2018): CFD investigation on steady and unsteady performances of contra-rotating propellers, Journal of Naval Architecture and Marine Engineering, Vol. 15, No. 2.

https://dx/doi.org/10.3329/jname.v15i2.36225

Kinaci, O. K., Gokce, M. K., Alkan, A. D. and Kukner, A. (2018): On self-propulsion assessment of marine vehicles, Brodogradnja / Shipbuilding Journal, Vol. 69, No. 4.

https://dx.doi.org/10.21278/brod69403

Kurobe, Y., Ukon, Y., Koyama, K., Makino, M. (1983): Measurement of cavity volume and pressure fluctuations on a model of the training ship "SEIUN-MARU', with reference to full scale measurement [in Japanese]. Ship Research Institute Technical, Report No. (NAID) 110007663078.

Launder, B. E., and Spalding, D. B. (1972): Lectures in Mathematical Models of Turbulence, Academic Press, London, England.

Menter, F. R. (1994): Two-equation eddy-viscosity turbulence models for engineering applications, AIAA Journal Vol. No 8 pp.1598-1605.https://dx/doi.org/10.2514/3.12149

Mosaad, M. A., Mosleh, M., El-Kilani, H. (2011): Guidelines for numerical flow simulation around marine propeller, First International Symposium on Naval Architecture and Maritime, Istanbul.

Nakatake, K., Ando, J., Kataoka, K. and Yoshitake, A. (2002): a simple surface panel method SQCM for ship hydrodynamics, IABM, International association for boundary element methods, UT Austin, TX, USA.

Prakash, S. and Nath, D. R. (2012): Computational method for determination of open water performance of a marine propeller, International Journal of Computer Applications, Vol. 58, No. 12.

Rhee, S. H., Joshi, S. (2005): Computational validation for flow around a marine propeller using unstructured mesh based Navier-stokes solver, JSME International Journal Series B, Vol.48 (3), pp.562-570.

https://dx.doi.org/10.1299/jsmeb.48.562

Seo, J. H., Seol, D. M., Lee, J. H., and Rhee, S. H. (2010): Flexible CFD meshing strategy for prediction of ship resistance and propulsion performance, International Journal of Naval Architecture and Ocean Engineering, Vol. 2, pp.139-145.

Ukon, Y., Kudo, T., Yuasa, H., and Kamiirisa, H. (1991): Measurement of pressure distribution on full scale propeller. In: Proceedings of the Propellers/Shafting '91 Symposium, the Society of Naval Architects and Marine Engineers, Virginia Beach, Virginia.

Ukon, Y., Kurobe, Y., and Kudo, T. (1989): Measurement of pressure distribution on a conventional and highly skewed propeller model under non-cavitating condition, Journal of the society of naval architects of Japan, Vol.165, pp.83-94.

Wang, C., Huang, H., Chang, X., and He, M. (2010): Applying periodic boundary conditions to predict open water propeller, Journal of Marine Sciences and Application, 9:262-267.

https://dx.doi.org/10.1007/s11804-010-1005-6

Watanabe, T., Kawamura, Y., Takekoshi, Y., Maeda, M., and Rhee, S.H. (2003): Simulation of steady and unsteady cavitation on a marine propeller using a RANS CFD code, Proceedings of the Fifth International Symposium on Cavitation, Osaka, Japan. 